\title{
Antarctic analog for dilational bands on Europa
}

\author{
T.A. Hurford ${ }^{\mathrm{a}, *}$, K.M. Brunt ${ }^{\mathrm{b}, \mathrm{c}}$ \\ a Planetary Systems Lab., NASA Goddard Spaceflight Center, Greenbelt, MD 20771, USA \\ b ESSIC, University of Maryland, College Park, MD 20740, USA \\ c Cryospheric Sciences Laboratory, NASA Goddard Space Flight Center, Greenbelt, MD 20771, USA
}

\section{A R T I C L E I N F O}

\section{Article history:}

Received 20 December 2013

Received in revised form 4 April 2014

Accepted 11 May 2014

Available online 27 June 2014

Editor: C. Sotin

We would like to dedicate this work in memory of Dr. B. Randall 'Randy' Tufts (1948-2002)

\section{Keywords:}

Europa

tectonics

Earth analog

Antarctica

\begin{abstract}
A B S T R A C T
Europa's surface shows signs of extension, which is revealed as lithospheric dilation expressed along ridges, dilational bands and ridged bands. Ridges, the most common tectonic feature on Europa, comprise a central crack flanked by two raised banks a few hundred meters high on each side. Together these three classes may represent a continuum of formation. In Tufts' Dilational Model ridge formation is dominated by daily tidal cycling of a crack, which can be superimposed with regional secular dilation. The two sources of dilation can combine to form the various band morphologies observed. New GPS data along a rift on the Ross Ice Shelf, Antarctica is a suitable Earth analog to test the framework of Tufts' Dilational Model. As predicted by Tufts' Dilational Model, tensile failures in the Ross Ice Shelf exhibit secular dilation, upon which a tidal signal can be seen. From this analog we conclude that Tufts' Dilational Model for Europan ridges and bands may be credible and that the secular dilation is most likely from a regional source and not tidally driven.
\end{abstract}

Published by Elsevier B.V.

\section{Introduction}

Lineaments are pervasive on Europa's surface. The majority of these features have been interpreted to form as a result of tensile failure (McEwen, 1986; Leith and McKinnon, 1996). This interpretation is supported by the fact that ice has a low tensile strength that ranges between about $100 \mathrm{kPa}$ and $2 \mathrm{MPa}$ (Lee et al., 2005) and that Europa naturally experiences tension from diurnal tidal stress that can meet this failure threshold (Greenberg et al., 1998). Moreover, some lineaments display dilational separation indicating that large extensions of the ice shell have occurred. The tidal cycle alone would not predict net extension so the second source of extension on either a regional or global scale is needed.

The most common lineament morphology are ridges or double ridges (Fig. 1a). These features consist of a trough that is flanked by a ridge on either side. They may extend $1000 \mathrm{~s}$ of $\mathrm{km}$ across the surface and are typically a few $\mathrm{km}$ wide and a few $100 \mathrm{~s}$ of $\mathrm{m}$ tall. Ridges represent an advanced state of an evolutionary development process (Geissler et al., 1998; Head et al., 1999). In its early stage, ridges are thought to form as tensile failure of the surface, forming a trough with no ridges. Subsequent processing of the crack then can build the observed ridge edifices with time.

There are a number of models for subsequent ridge formation that have very different implications for fundamental processes

\footnotetext{
* Corresponding author.

E-mail address: Terry.A.Hurford@nasa.gov (T.A. Hurford).
}

on Europa. However, all assume the formation of the tensile fracture but then differ on the ridge building mechanism. Kadel et al. (1998) builds the ridges through fissure eruptions of material from the crack onto the surface adjacent to the fissure, while Head et al. (1999) describes upward bending of the surface due to a buoyant upwelling of ice intruding along the fissure. Turtle et al. (1998) incremental wedging model forms ridges from compression of material that gets trapped in the fissure and drives deformation on each flank. Finally, Greenberg et al. (1998) ridge model appeals directly to the working of the fissure by tidal stresses to expose, freeze and extrude material onto the surface as the fracture opens and closes daily.

While Greenberg et al. (1998) ridge formation model acknowledges the role of tidal processing in ridge formation, all the other models might also be linked to the cyclical tidal processes that are thought to work on active fractures on Europa (Prockter and Patterson, 2009). For example, fissure eruptions might be controlled by tidal stress (Hurford et al., 2007), tidal shear stress might provide a source of heat that drives buoyant upwelling ice (Gaidos and Nimmo, 2000) or tidal stress might open a crack allowing material to slump into the fissure, which later, when compressed by tidal stress forms wedge ridges. Therefore, the role of diurnal tidal stresses may be critical to the formation of ridges on Europa.

Apart from ridges, extensional features known as dilational bands are observed on Europa. Bands are widely distributed on Europa's surface and can be a few $10 \mathrm{~s}$ of km across, although this width may not always be uniform (e.g. wedged shaped bands) and 

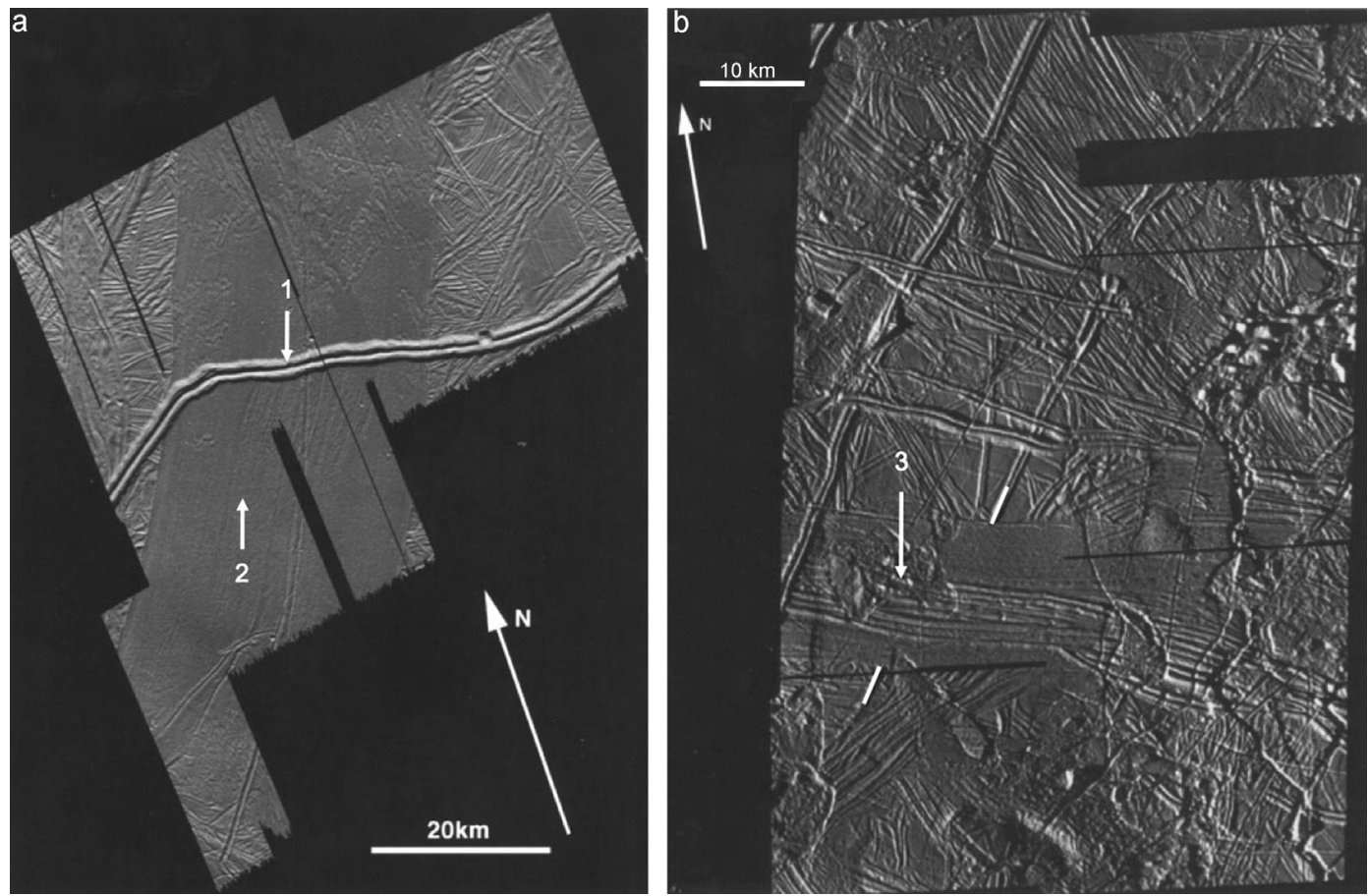

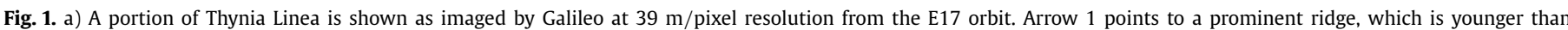

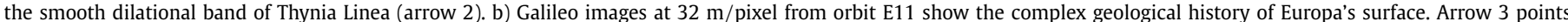

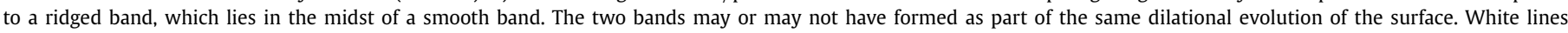
on either edge of the band show piercing points for reconstruction purposes (cf. Tufts et al., 2000).

can extend $100 \mathrm{~s}$ of $\mathrm{km}$ in length. Evidence for the extensional nature of these bands comes from the fact that ridge terrain on either side of the band can be matched up with the removal of the band infill material in reconstructions (Schenk and McKinnon, 1989; Sullivan et al., 1998). This fact implies that band interiors are composed of new material relative to the surrounding terrain (Prockter et al., 2002). The interior structure of the bands can range from relatively smooth fine striae, aligned with the band's margins (Fig. 1b) or the more coarse structures (such as Ridged Bands), which resemble the edifices that comprise ridges (Fig. 1c).

Some dilational bands show evidence of raised flanks (see below), which implies that extension leading to the band exploited preexisting fractures in the crust. While the regional extensional stress needed for the dilational band may not necessarily be responsible for the initial failure of the surface nor participate in the ridge building process, this extension may be contemporaneous with daily diurnal tidal stresses, which participate in the ridge building process. Tufts et al. (2000) explored the interplay between regional extension and diurnal tidal process to define a morphological continuum of evolution from ridges to smooth dilational bands.

\subsection{Tufts' Dilation Model}

In Tufts' Dilation Model, ridges represent one end member of the formation continuum. In the model, diurnal tidal stresses work a fracture, slowly building ridges on its flanks. There is no regional dilation to affect this process (Fig. 2, case a). While Tufts et al. (2000) originally linked their model to Greenberg et al. (1998) ridge formation model, this is not a rigid constraint on Tufts' Dilation Model. In fact, any of the above ridge-formation models are valid with Tufts' Dilation Model as long as ridge building is mainly influenced by diurnal tidal stresses or motions.

Once the daily cycle of stress acting on a fracture experiences regional extension concurrent to the ridge building process, a dilational band will be formed. Its morphology will depend on the interplay between the ridge building process and the regional extension. This interplay depends on the "dilational quotient", which is defined as the ratio of the rate of the regional secular dilation to the cyclical diurnal tidal dilation. If the quotient is very low, ridges forms without much dilation. The formation of smooth bands is the other end member of Tufts' Dilation Model (high quotient), where dilation dominates over ridge growth. As long as the dilation quotient remains uniform over the timescale of band formation, a band with a smooth texture or fine striae will be formed (Fig. 2). However, the magnitude of the dilation quotient can affect the relief of the band infill material compared to the surrounding terrain. If the dilational quotient is low, the band is spreading out the ridge building process, leading to a smooth band whose infill terrain stands at the height of a typical ridge (Fig. 2, Case b). But if the dilational quotient is high, no ridge building can occur, leading to a smooth band whose infill terrain stands at the height of the background terrain or lower (Fig. 2, Case c).

More coarse dilational bands, such as ridged bands, may form from regional extension, which is nonuniform over the timescale of band formation. Ridged bands can form in Tufts' Dilation Model as a band dilates with episodes of no (or slow) secular dilation interspersed with times of high secular dilation. For example as shown in Fig. 3, a ridge may form along a crack during a period of no (or slow) secular dilation (a), but then rapid dilation can spread apart the ridge pair, creating a uniform floor (b) upon which, once dilation slows or ceases, a new ridge pair can form (c), until the next episode of rapid dilation. Thus, over time and cycles of slow and rapid dilation, a band of ridges can form (Fig. 3d).

Using Tufts' Dilational Model, examples of band formation on Europa can be described in terms of the processes of secular and tidal dilation. Fig. 4 shows the location of the roughly $10 \%$ of Europa's surface that has been mapped at about $250 \mathrm{~m} /$ pix resolution to study the geology on its surface. Within this region, the location of 10 examples of bands have been called out and insets of the bands themselves included. The formation of these bands can be described in the context of Tufts' Dilational Model. 


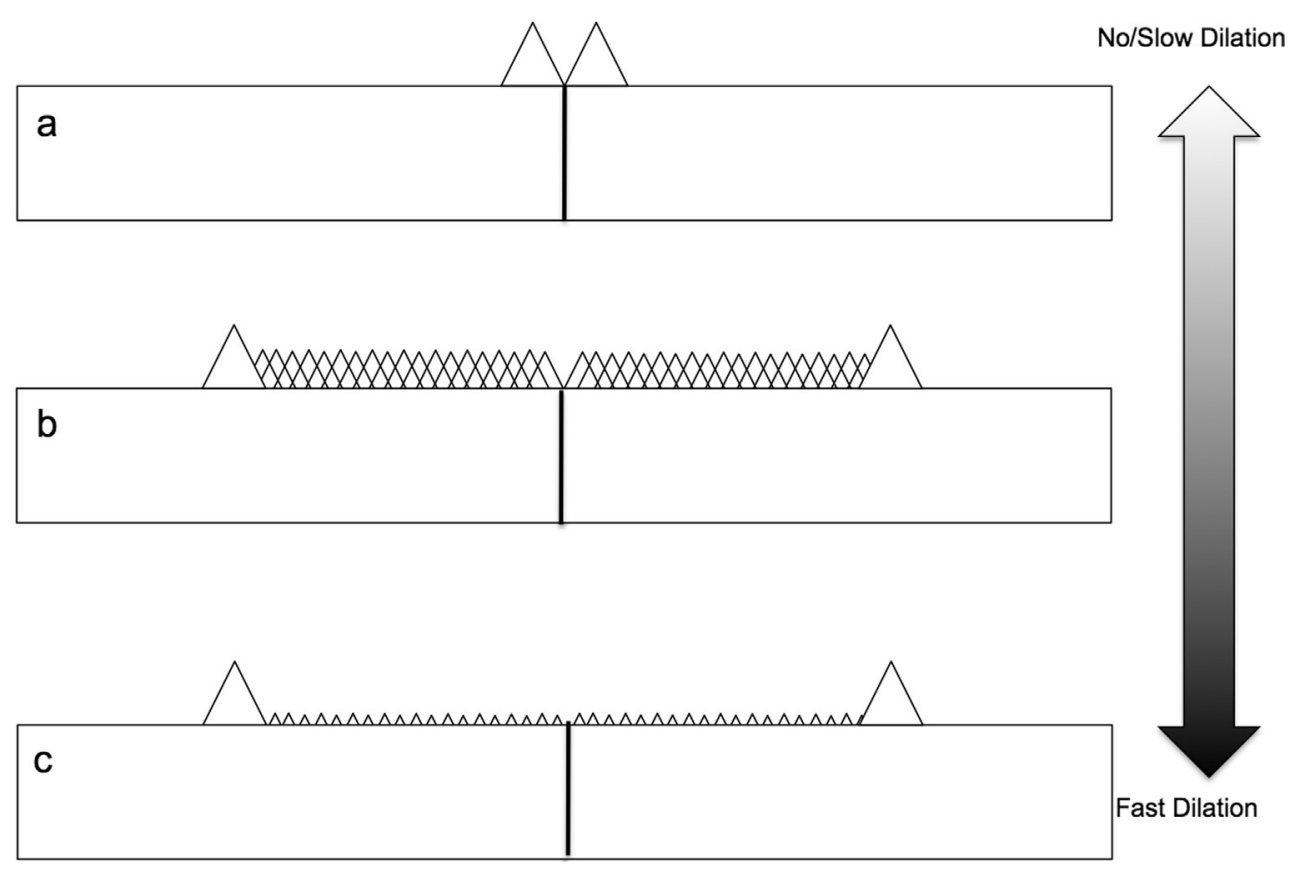

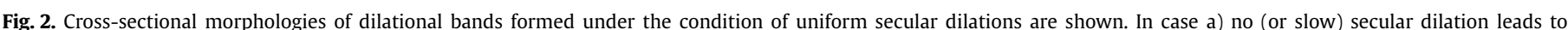

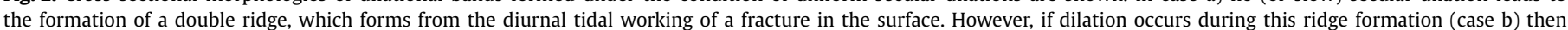

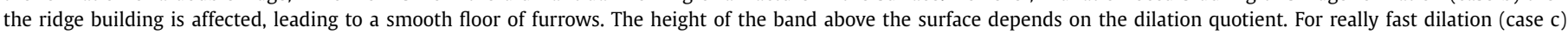

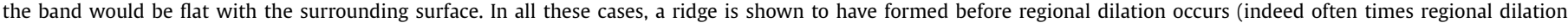

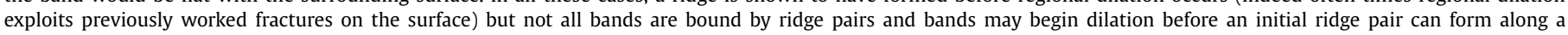
fracture.

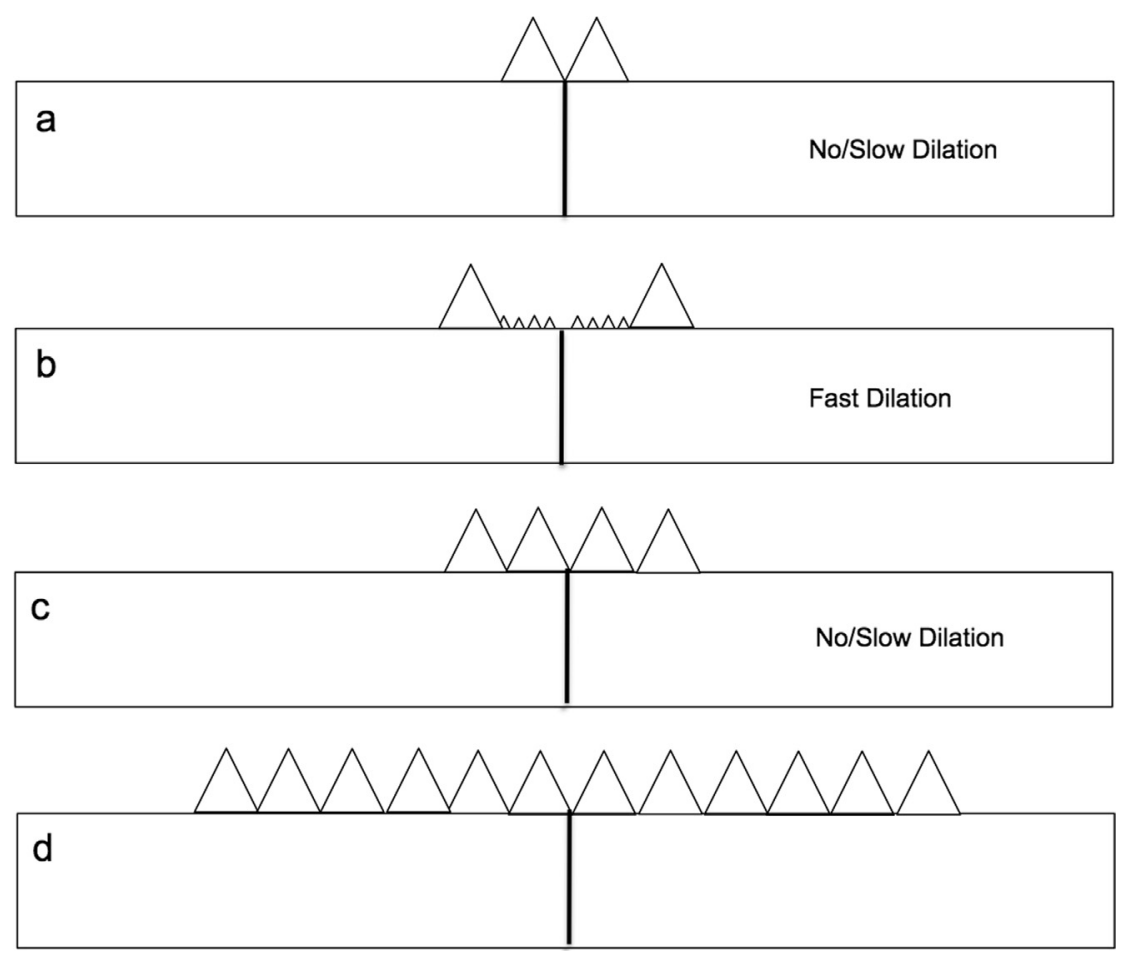

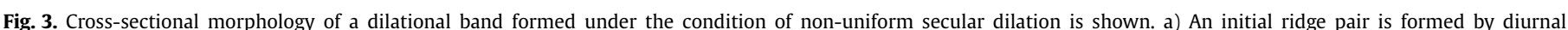

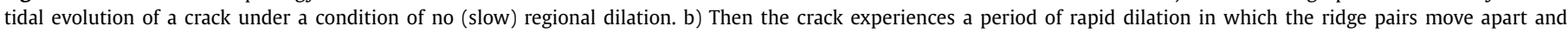

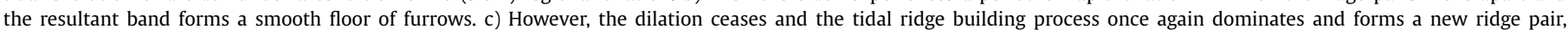
overprinting the smooth band floor interior to the original ridge pair. d) This process may repeat in regular episodes of secular regional dilation and tidal ridge building.

For Cases \#1-5 in Fig. 4a the secular dilation that formed the bands was very uniform over the timescale of their formation. This is evident by the smooth texture, at these resolutions, of the interior material in these bands. In some cases (Cases \#2 and \#4), ridges formed at a time when no secular dilation in the region was occurring. Then after ridge formation, uniform secular dilation spread the ridges apart, producing a smooth band, bounded by the remnant ridge pair. In Cases \#3, \#4, and \#5, dilation exploited a crack before ridge formation could occur. In these cases, a smooth band without ridge margins are formed. 
a

Bands formed under Uniform Dilation
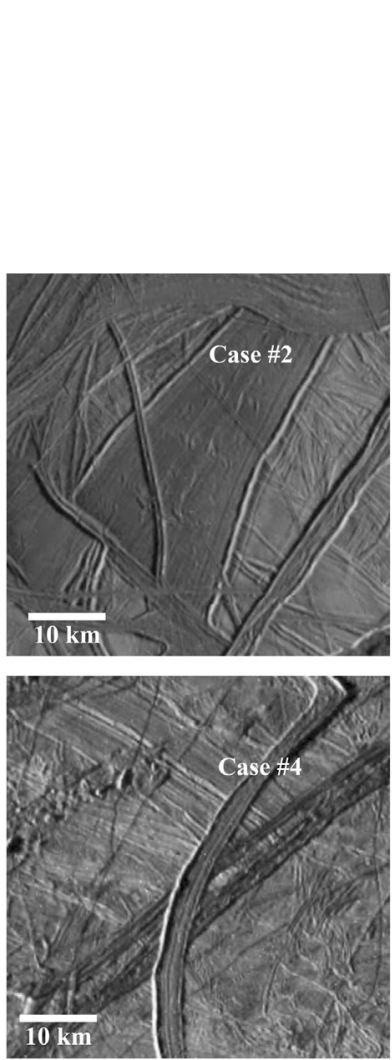

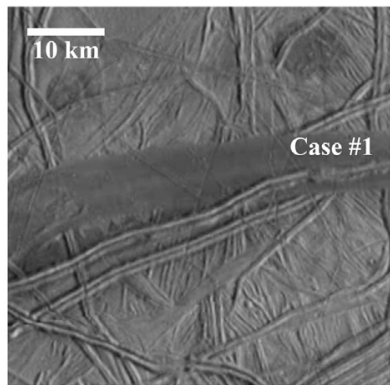

b

Bands formed under Non-uniform Dilation
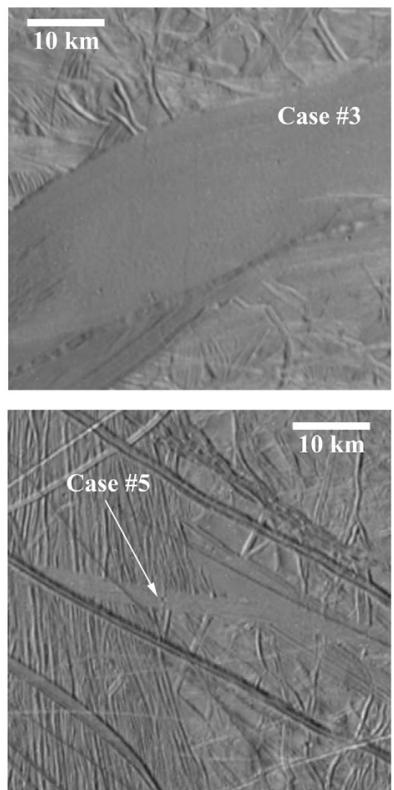
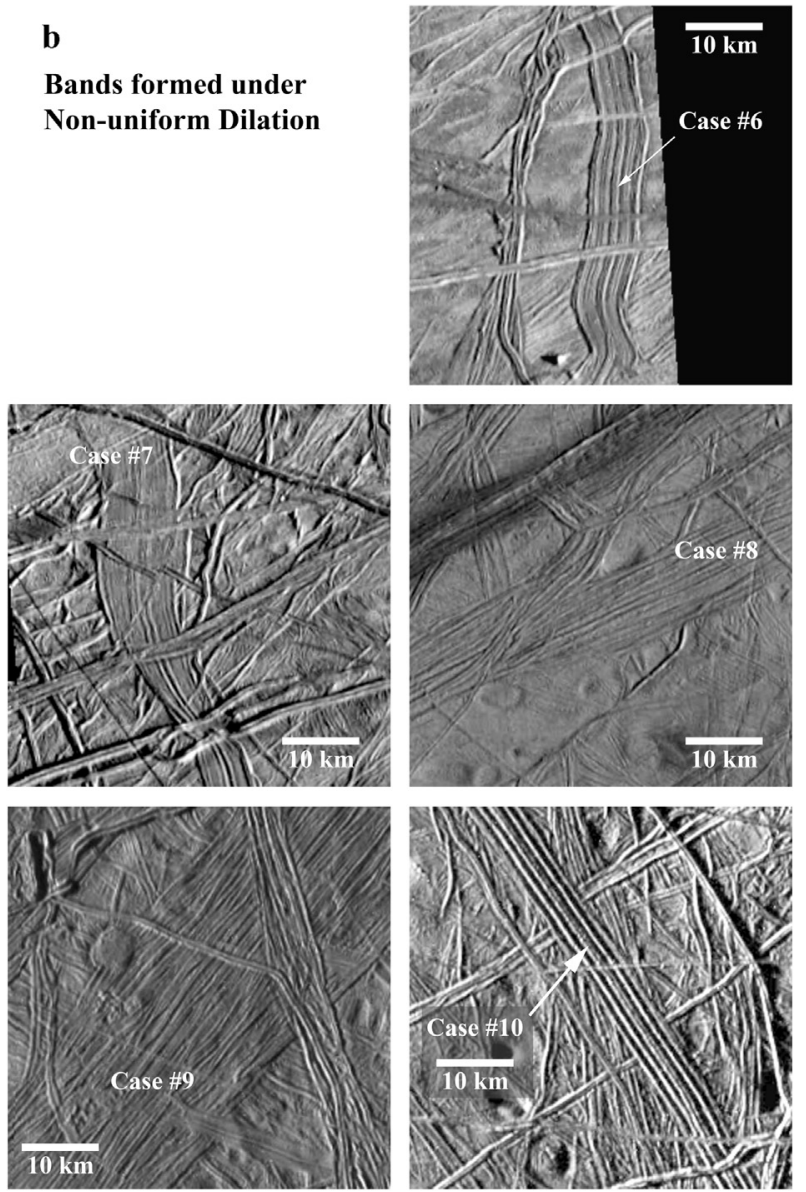

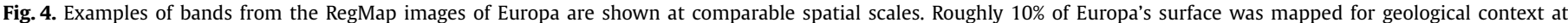

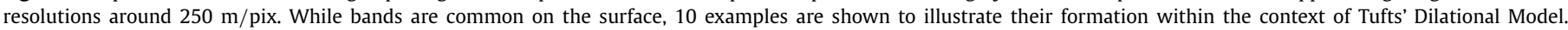

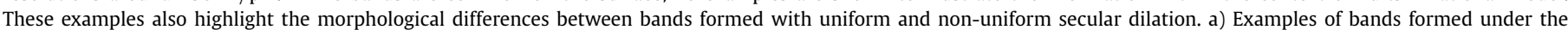

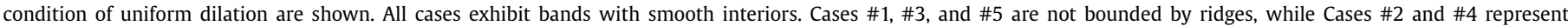

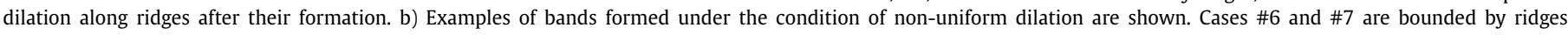

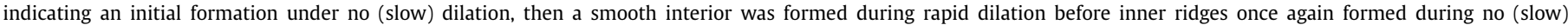
dilation. Cases \#8, \#9, and \#10 represent many cycles of no (slow) dilation interspersed with rapid dilation.

More coarse bands (Fig. 4b, Cases \#6-10) form according to this model when secular dilation is non-uniform during the band formation timescale. In Cases \#6 and \#7, a ridge pair forms before secular dilation begins. Once this initial pair was established uniform, relatively quick secular dilation spread the ridge pairs apart, forming a smooth interior band. However, the secular dilation did not remain constant and at some point slowed or stopped, allowing a ridge pair to form down the center of these bands. In Cases \#8 and \# 9, many episodes of no (or slow) secular dilation was experienced, producing ridge pairs, which were separated during times of rapid secular dilation, spreading these pairs. Once the ridge pairs were separated, dilation slowed to allow an interior ridge pair to form. The numerous sets of parallel ridges indicate the secular dilation underwent many periods of rapid and slow dilation.

Fig. 4b, Case \#10 represents about three cycles of rapid and slow secular dilation. The band appears to be made of 5 raised ridges. Its formation started with the development of a ridge pair during a time of no (or slow) secular dilation. Then secular dilation increases and this ridge pair separated. After a time the secular dilation slowed or stopped and once again a ridge pair was formed interior to the original ridges. Another period of rapid secular dilation occurred and then slowed. The final ridge pair then formed in the very center. Although, it looks like 5 raised ridges, the very central ridges has a very subtle trough running down its center and is actually a ridge pair. Thus, the band represents the formation of 3 ridge pairs, formed during secular dilation to form this ridged band.

Although Tufts' Dilation Model can conceptually explain the morphologies of tectonics from ridges to bands seen on Europa, much remains uncertain: The exact process by which tidal stresses work an existing fault is not understood in detail. Moreover, the mechanism driving regional or global secular dilation is unknown. The conceptual model assumes tidally driven offsets that are small and driven on the diurnal timescale, yet no direct observations of this motion on Europa are possible and can only be inferred in the tectonic record. However, we can verify the plausibility of Tufts' Dilational Model through identification and analysis of an Earth analog.

Early analogs for Europan surface morphologies focused on terrestrial sea ice, or the thin frozen layer of the ocean surface (Pappalardo and Coon, 1996; Greeley et al., 1998). These studies included comparisons of patterns of deformation. Deformation of sea ice results primarily through wind and wind-driven ocean-surface currents. To assess Tufts' Dilation Model, which is dominated by tidal cycling of a fracture, we turn our attention to an Antarctic ice shelf, where steady, viscous ice flow has been shown to be strongly effected by tidal forcing (Brunt, 2008; Brunt et al., 2010b; King et al., 2011; Makinson et al., 2012; Brunt and Macayeal, 2014). 


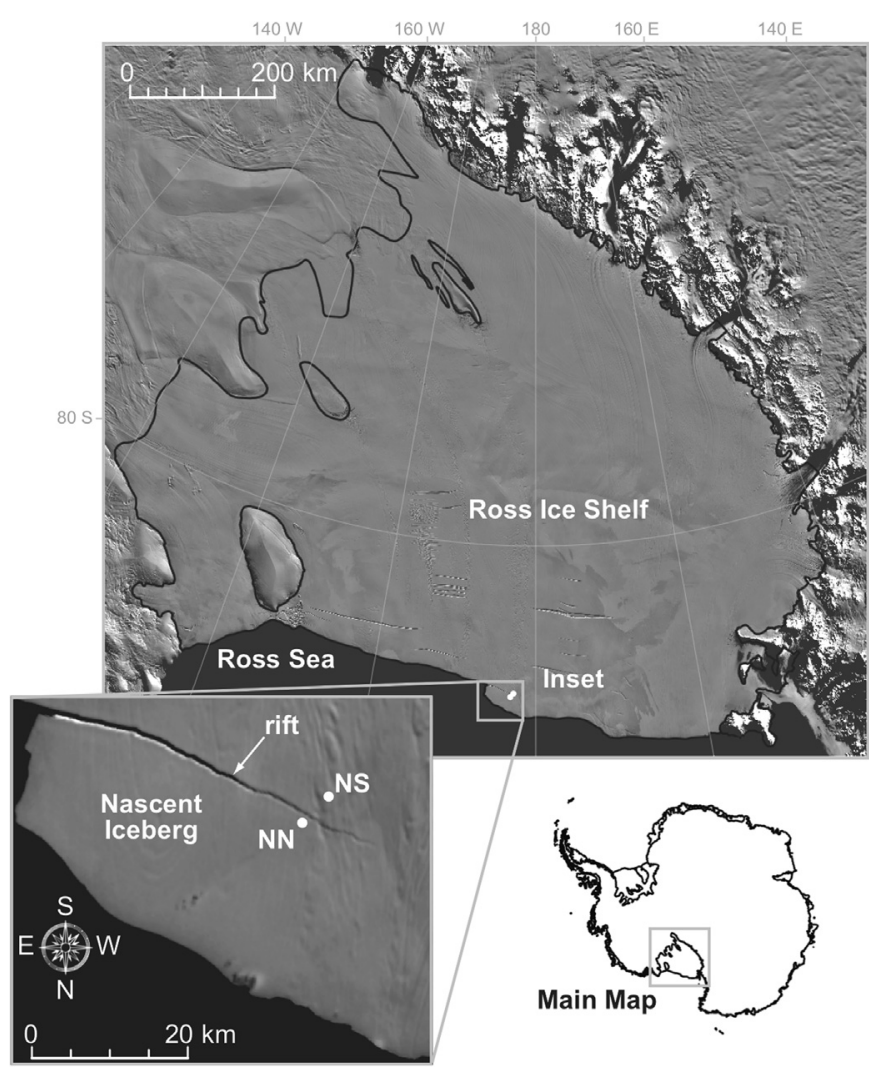

Fig. 5. GPS stations at Nascent Iceberg, on the Ross Ice Shelf, on the 2004 MODIS mosaic (Scambos et al., 2007). Stations NN and NS were surveyed in 2005; their locations on this map were migrated to their 2004 positions based on the InSAR velocity data of Rignot et al. (2011).

GPS data from 2005 taken at stations on either side of a rift on the Ross Ice Shelf, Antarctica, revealed that each station recorded daily tidal motions (Brunt et al., 2010b). In this paper we reexamine the GPS data to explore tidally driven motions along a rift on the Ross Ice Shelf, Antarctica that are analogous to tidally driven motions experienced on Europa. And we explore the interplay between diurnal dilation with regional secular dilations on the rift.

\section{A rift on Ross Ice Shelf, Antarctica}

Ice shelves are large regions of floating ice that extend from land out over the open ocean. Around the coast of Antarctica they are on the order of $100 \mathrm{~s}$ of $\mathrm{m}$ thick. They generally form in protected bays on the continental shelf. The water column beneath the floating ice-shelf is also on the order of $100 \mathrm{~s}$ of $\mathrm{m}$ in depth. Approximately 5-10 $\mathrm{km}$ downstream from where the ice sheet loses contact with the underlying bedrock, ice shelves are hydrostatic, and are fully compensated by buoyant force (Brunt et al., 2010a). As such, they respond to the ocean tide, with their surface elevation changing with the full range of the tidal amplitude. On the Ross Ice Shelf, the vertical tidal range is typically 1-2 m but can be as much as $3 \mathrm{~m}$ at its southern limit, near the grounding zone (Padman et al., 2003). In Antarctica, with respect to ice-mass balance of the continent, the bulk of ice-mass loss occurs through ice shelves in the forms of iceberg calving and basal melt (Rignot et al., 2013).

On the Ross Ice Shelf (Fig. 5), which is the largest Antarctic ice shelf at nearly $500,000 \mathrm{~km}^{2}$, a rift has propagated from a preexisting notch in the ice-shelf calving front. This rift is a discontinuity that extends vertically through the ice shelf and is presumed to be continuing to propagate westward, eventually connecting again with the ice front to create a new 20 by $40 \mathrm{~km}$ iceberg. Nearly the entire 40-km length of the rift is partially filled with a mélange of snow, firn, and marine ice (Leonard et al., 2008) (Fig. 6). However, the surface of the mélange is $\sim 30 \mathrm{~m}$ below the surface of the ice shelf (Fricker et al., 2005) and processing of the material within the mélange contributes to rift widening (Leonard et al., 2008). Currently, as part of the natural ice-shelf calving process, this rift widening is contributing to the westward rift propagation that will likely lead to a large iceberg-detachment.

As part of a study of ice-shelf flow, kinematic GPS data from this Antarctic site, named Nascent Iceberg, was collected at multiple stations (see Fig. 5, inset), including two referenced as Nascent North (NN) and Nascent South (NS), in November 2005 (Brunt et al., 2010b). These two GPS stations were located on either side of the Nascent Iceberg rift system, separated by $\sim 4.2 \mathrm{~km}$. At each station, a dual-frequency GPS receiver sampled data continuously every $15 \mathrm{~s}$. Both stations collected data for a minimum of 16.9 days.

The nearest permanent GPS reference site was more than $300 \mathrm{~km}$ away. Hence, the data were analyzed using a kinematic Precise Point Positioning approach, using the software package GIPSY, as outlined by Brunt (2008) and Brunt et al. (2010b). The data taken from each station were processed separately to quantify how fast each station was moving. Specifically, the motion of each station was detrended, to remove the net rate of displacement, and then separated into northing, easting, and vertical components. The data were then filtered using noise uncertainty values, as determined by GIPSY, of $>0.1 \mathrm{~m}$ for the vertical and $>0.05 \mathrm{~m}$ for the easting and northing time series. Finally, the data were smoothed to eliminate short-term transient motion, both real and associated with instrument error, with periods $\leq 15 \mathrm{~min}$.

Brunt et al. (2010b) found that the residual horizontal motion was diurnal and varied in amplitude over the $\sim 14$ day period, correlating with solar and lunar tidal forcing (cf. Brunt et al. (2010b), Fig. 2).

\section{Analog for band formation on Europa}

Brunt (2008) provides an extensive tidal analysis of the Nascent Iceberg GPS data. For that study, a least-squares method of fitting linear and harmonic terms was used to extract motions of the ice shelf that varied at tidal periods. The study focused on 8 major tidal constituents, including solar $\left(\mathrm{P}_{1}\right)$ and lunar $\left(\mathrm{K}_{1}, \mathrm{O}_{1}\right.$, and $\left.\mathrm{Q}_{1}\right)$ diurnal constituents and solar $\left(\mathrm{S}_{2}\right)$ and lunar $\left(\mathrm{M}_{2}, \mathrm{~N}_{2}\right.$, and $\mathrm{K}_{2}$ ) semi-diurnal constituents. The results indicated that diurnal constituents (specifically the lunar $\mathrm{K}_{1}$ and $\mathrm{O}_{1}$ constituents, with periods of 23.93 and $25.82 \mathrm{~h}$, respectively) dominated the observed motion at Nascent Iceberg, in both the horizontal and vertical directions.

Based on a least-squares analysis of the 16.9-day observational period, Brunt (2008) reported time-mean horizontal velocities for GPS stations NN and NS of $1094 \mathrm{ma}^{-1}$ and $1070 \mathrm{ma}^{-1}$, respectively. Some of the difference between the time-mean horizontal velocities of the two stations was related to differences in ice thickness, which governs the rate of viscous ice flow. However, given the short ( $\sim 4.2 \mathrm{~km}$; Fig. 5) distance between the stations, we assume that the bulk of the difference was associated with the rate at which the rift was widening.

To investigate the rift-widening of the stations, the data from NN and NS were detrended, filtered, and separated into northing, easting, and vertical components, following Brunt et al. (2010b). The data were then linearly interpolated to a common timeline to determine the separation rates between the stations throughout the observational period. As shown in Fig. 7a, the overall net distance between the stations was increasing with time. During this 16.9-day period the net distance, or predominantly, the rift widening between the 2 stations, amounted to $\sim 1.5 \mathrm{~m}$, with a widening rate (calculated using a least-squares method) of $0.09 \mathrm{mday}^{-1}$ 


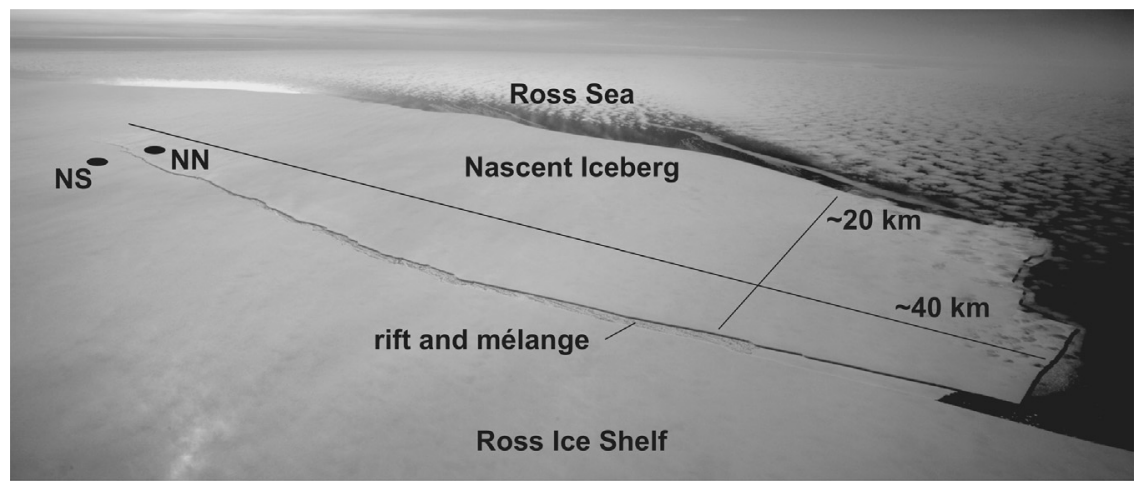

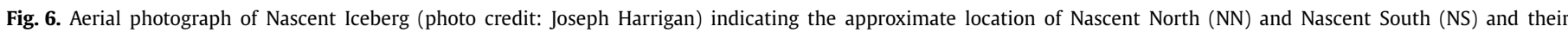
relation to the rift system.
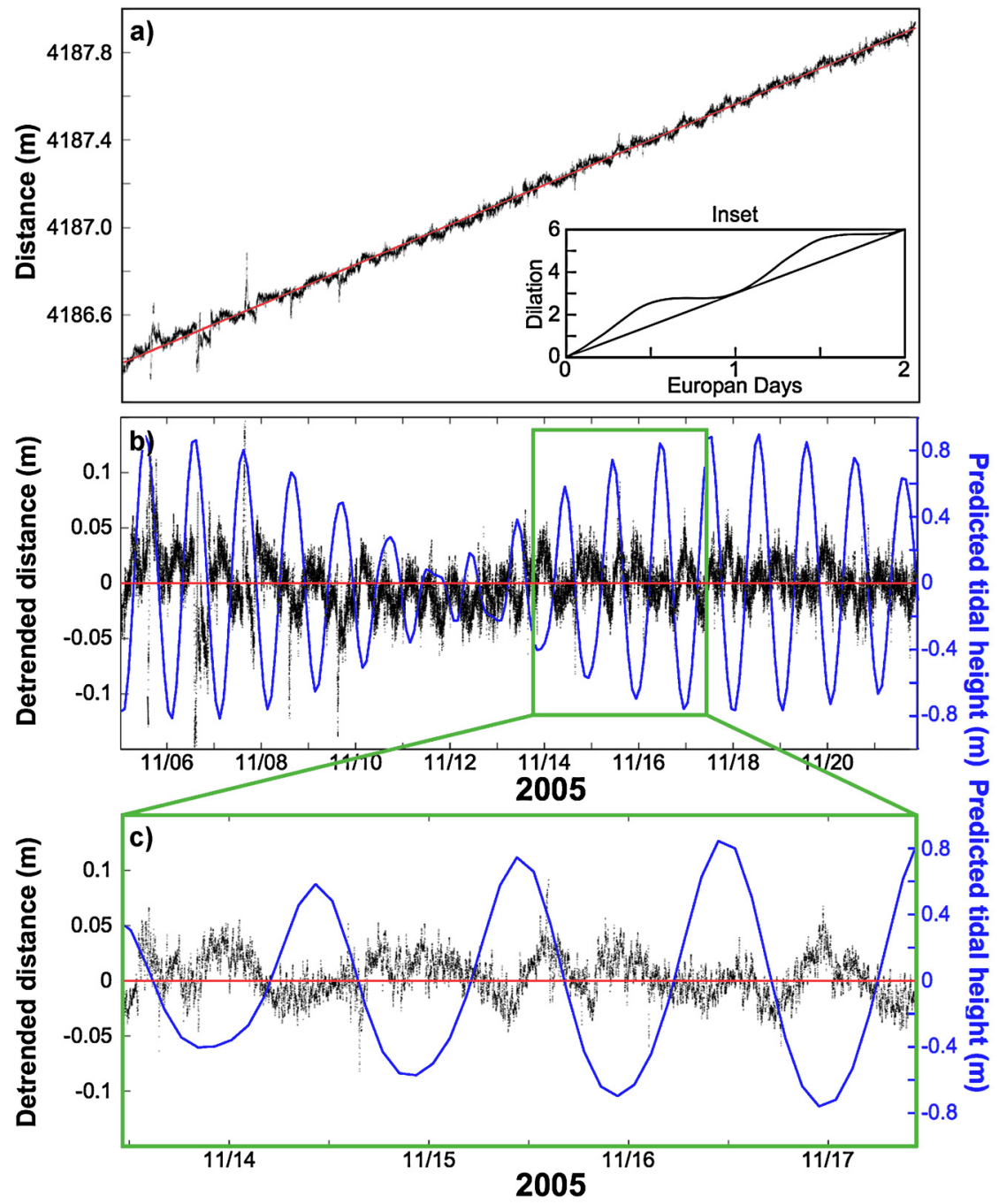

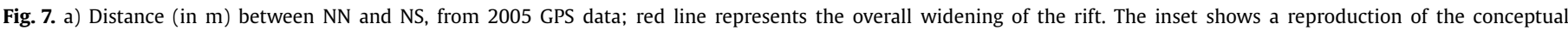

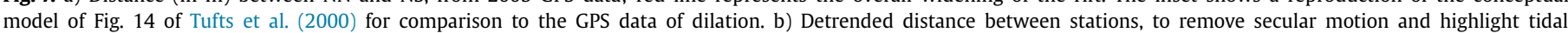

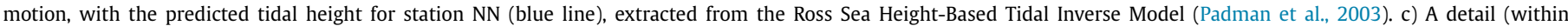
the green box in Fig. 5b) of the detrended distance between stations and predicted tidal height for station NN.

(33.0 $\left.\mathrm{ma}^{-1}\right)$. This rate of motion was used to remove the secular motion between the two stations, in order to highlight the tidally induced motions.

The GPS time series from each station on either side of the Ross Ice Shelf rift system indicated that the rift-widening rate was not constant. While there is an overall secular motion (Fig. 7a, red line), there is also a superimposed cyclical tidal motion (Fig. 7a, black points). Small diurnal motions are cyclically amplifying and diminishing the secular motion, suggesting that the rift-widening is affected by tidal forcing. Removing the secular motion highlights the tidally induced motion (Fig. $7 \mathrm{~b}$ is a detrended version of Fig. 7a, with the secular motion removed, to highlight the 
cyclical motion). For comparison, Figs. 7b and 7c include the predicted tidal height for station NN, which was extracted from the Ross Sea Height-Based Tidal Inverse Model (Padman et al., 2003), based on GPS position and time of acquisition. From these figures, it is apparent that the period of maximum horizontal separation correlates with the diurnal tidal height minimum.

\section{Discussion}

The Antarctic rift displays daily tidal motions, which are the result of the tidal stress in the Ross Ice Shelf. Maximum horizontal separation, driven by lateral tidal motions, correlates with the diurnal tidal height minimum. This implies that lateral motion is not simply driven by expansion of the ice sheet due to higher ocean tides. Moreover, the tidal motions exist even though the ice shelf is fractured. Tidal stress in Europa's ice shell are computed as if the shell weren't fractured, while it is not clear if the presence of fractures would relieve this stresses preventing tidal motions, the Ross Ice Shelf example shows that the presence of a rift does not prevent tidal stress from driving motion along its length. Thus, the assumption that tidal stress can drive motion along cracks on Europa appears valid. This also has important implication for tidal control of eruptions on Enceladus (Hurford et al., 2007).

The interaction between tidal dilation and secular dilation is consistent with the conceptual model of band formation on Europa as envisioned by Tufts' Dilational Model, as can be seen by comparing Fig. 7a with Fig. 14 from Tufts et al. (2000) or the reproduction of those results as shown in the inset of Fig. 7a. In both the GPS data from Antarctica and Tufts' Dilational Model, the widening of the rift from regional secular dilation is modulated by the tidally driven motions. In Tufts' Dilational Model, tidal motions can reinforce the secular dilation, leading to larger amounts of dilation, but when tidal motions work against the secular dilation the net dilation returns to the net secular offset (inset of Fig. 7a). However in the GPS observations, when tidal motions retard secular motions, they overcome these secular motions, producing net offsets that are less than the purely secular offset (Fig. 7a). The possibility that tidal motion can overcome secular dilation wasn't included in the conceptual Tufts' Dilational Model and is a minor adjustment that should be invoked in future model applications.

In Tufts' Dilational Model, the dilational quotient used to determine if secular dilation dominated the tidal dilation was defined as a ratio of dilational rates. Based on the observation of tidal motions on the Ross Ice Shelf rift we conclude that this quotient, while a useful concept, needs modification. While it is straight forward to evaluate the secular dilation rate, the tidal dilation rate is problematic. We propose that the dilational quotient be the ratio of the secular dilation over one tidal cycle to the amplitude of tidal dilation. In the Antarctic case this value would be $\sim 2$ since the secular dilation is $0.09 \mathrm{~m}$ over one tidal cycle (a day) and the rift dilates with an amplitude that is a little less than $0.05 \mathrm{~m}$. At quotient values $\sim 6$, a rift would never experience a net compression, as even when tidal stresses are compressive, the secular dilation still results in net tension on the rift. Quotients $>6$ would be considered high (Fig. 2c), most likely leading to smooth band morphologies on Europa, while values $<1$ would be low, allowing some tidally controlled ridge building to occur. Values in the 1-6 range would produce hybrid morphologies such as smooth bands with floors above the ambient terrains (Fig. 2b).

From the secular motion along the Antarctic rift, the viscous strain rate was found to be $\dot{\varepsilon}=1.5 \mathrm{~m} /(4187 \mathrm{~m} \cdot 16.9$ days $) \approx$ $2.5 \times 10^{-10} \mathrm{~s}^{-1}$, which yields a viscous stress of $\sigma_{v}=\eta \dot{\varepsilon} \approx$ 0.25 bars, if the viscosity, $\eta$, of the ice shelf flow is $\sim 1 \times 10^{14}$ Pa s as modeled by Brunt (2008); Brunt and Macayeal (2014). From the cyclical tidal motions, the peak elastic strain can be found from the amplitude of the tidal motion and the distance between the stations, $\varepsilon=0.05 \mathrm{~m} / 4187 \mathrm{~m} \approx 1.2 \times 10^{-5}$. In order for the elastic tidal stresses to overcome the viscous stress and constrict the rift, the elastic stress has to be comparable if not larger than the viscous stress. If Young's modulus of the mélange was known, a direct comparison could be made, but the mélange cannot be sampled as researchers are not permitted to explore the rift's interior. However, we predict that Young's modulus of the mélange must be at least $\approx 2 \times 10^{9} \mathrm{~Pa}$, which implies that it is similar to competent ice $\sim 5 \times 10^{9} \mathrm{~Pa}$ (Gudmundsson, 2011).

On Europa, tidal stress may aid in forming fractures on its surface and if the dilation experienced during phases of tension is not completely balanced by the contraction during phases of compression, it may lead to small amounts of net offset. However, diurnal tidal stress is not the source of regional dilation that forms bands, which exhibit large amounts of dilation. As is the case in Antarctica, larger viscous deformation of the ice shelf is the source of the regional stress causing the rift to dilate. On Europa, larger movements of the ice crust could also provide a source of regional dilation. But diurnal tidal motions can be comparable in magnitude to daily secular dilation and the interplay between the two motions could be important in controlling the morphologies of the bands that form under the influence of the regional dilation, just as proposed for Europa by Tufts et al. (2000).

On Europa, tidal stress is on the order of 1 bar and if the cold ice shell has a Young's modulus of $5 \times 10^{9} \mathrm{~Pa}$, then Europa fractures could experience strain on the order of $\sim 2.0 \times 10^{-5}$. This level of strain implies that Europan fractures have tidal dilation amplitudes on order of $10 \mathrm{~cm}$. Bands such as Thynia Linea (Fig. 1a) display $27 \mathrm{~km}$ of dilation and may be classified as having a high dilational quotient and close to that end member of band morphology (Tufts et al., 2000). Therefore, assuming a tidal dilational amplitude of $10 \mathrm{~cm}$ and a high dilational quotient of 6 , secular dilation along Thynia could be $60 \mathrm{~cm}$ per orbit or $\sim 17 \mathrm{~cm} \mathrm{day}^{-1}$. At this rate Thynia could have formed in a maximum of 435 years. of course it may have experienced even higher dilational quotients, which would yield even shorter formation times. But even if formation is 1000 years or less (corresponding to dilational quotients of $>2$ ), this timescale is geologically quick, probably much quicker than the process of ridge formation and at least 100 times quicker than other models of band formation based on Earth analog spreading centers (Stempel et al., 2005). If indeed the source of secular dilation is related to viscous flow of the ice shell and this rate of formation is plausible, this model could be used to constrain the viscosity and thus, temperature of the ice-shelf driving secular motions.

Because both secular and diurnal tidal motions were observed on the Ross Ice Shelf rift, it may be a good analog for the interplay between secular and diurnal tidal motions experienced on Europa during band formation. However, the analog may only be applied to tidal and secular motions. Indeed, we would not expect ridge formation to occur along this Antarctic rift, as the environmental setting is very different from the Europan conditions. For example, if rift dilation on Europa were to expose volatiles such as liquid water, rapid boiling would occur since it does not posses an atmosphere. This might be one mechanism for ridge building, but would not be expected to operate in the Antarctic. Also, Earth's atmosphere causes weathering along the rift on the Ross Ice Shelf, which drives snowfall, and slumping of material into the rift, whose interior is described as a mélange. On Europa no atmospheric weathering processes are operating and the interiors of these bands are thus different, but slumping and freezing of material in the forming bands on Europa may also contribute to their widening (Greenberg et al., 1998) just at the mélange in the Antarctic rifts promotes secular widening.

In Antarctica, the rift fractures the ice shelf down to the liquid ocean beneath it. It's not clear if this condition is needed to 
enhance tidally driven motions; on Europa tidally active cracks most likely do not penetrate to a liquid ocean. However, on Europa it may be likely that cracks penetrate the elastic lithosphere, which may be less than a $1 \mathrm{~km}$ thick at fracture locations (Hurford et al., 2005; Billings and Kattenhorn, 2005). Thus, fracturing to a more ductile ice or liquid decoupling layer may be key to tidally driven motions. Antarctic fractures that have not fully formed into rifts in the ice shelf may be the key to testing this characteristic of the analog model.

On Europa, the ice shell does not present obvious free surfaces to accommodate tidally driven motions. On Earth, the Nascent ice berg terminates along a free boundary of the open ocean. This fact may allow tidally driven motions to be enhanced since these forces are pushing on the iceberg, which has room to shift, displacing it into the open ocean. Again, ice rifts or fractures in ice shelves, removed from the sea boundary, may allow the further improvements into our understanding of these analog sites. The affect of this free boundary might be a more important issue for tidally driven shear motions. Competing models of strike-slip motion on Europa, driven by tidal shearing, are sensitive to the parameterization of the domain boundaries. One strike-slip model is based on strike-slip motion along Earth continental plates (Smith-Konter and Pappalardo, 2008) in which a free boundary allows slip to occur differently than in a model in which motion must be allowed in a deforming shell (Rhoden et al., 2012). However, the GPS data was limited in the ability to decern shear motion and future study is warranted to address this difference.

Finally, while Tufts' Dilational Model captures the interplay of secular and tidal motions on the working of fractures, there are aspects of the model that must still be worked out in order to fully understand the formation of bands and the morphologies they exhibit on Europa. Tufts' Dilational Model was conceived in the context of a ridge building scenario developed by Greenberg et al. (1998), it may not be applicable only to that type of ridge formation scenario. Thus, the ridge building mechanisms should be recast in the framework of secular and daily tidal motions. Doing so might help develop these models and lead to better understanding of the ways in which ridges can be formed along cracks on Europa.

\section{Conclusions}

As demonstrated in the GPS data, an evolving rift near the calving front of the Ross Ice Shelf exhibits both secular widening and a cyclical, diurnal motion associated with tidal forcing. The Ross Ice Shelf rift system thus represents a useful analog for further modeling of the role of tidal motion in band formation on Europa. Moreover, Tufts' Dilational Model, which defines the link between ridges and bands, appears relevant to both Earth and Europa and is worthy of further investigation.

Furthermore, tidal motions observed along the Ross Ice Shelf rift might be important to study in order to understand other aspects of tidal motion along cracks on icy satellites. For example, rifts on Enceladus are the source locations for active cryovolcanism in its south polar region (Porco et al., 2006; Spencer et al., 2006; Spitale and Porco, 2007). Study of tidal stresses on these rifts has led to a Tidal Venting Model in which the timing and location of eruptions are controlled by tidal stresses (Hurford et al., 2007). While the tidal motions invoked in the Tidal Venting Model cannot be directly detected by orbiting spacecraft such as Cassini, study of similar motions along rifts in Antarctica may provide the best test for the tidal motions assumed by the Tidal Venting Model.

\section{Acknowledgements}

We thank NASA Headquarters for funding through the Science Innovation Fund (Surface Deformation of Icy Moons: Insights from Earth Analogs and Modeling). We thank Douglas MacAyeal and the NSIDC for Nascent Iceberg GPS data. We thank Jeanne Sauber, Alyssa Rhoden, Wade Henning, Ryan Walker and Tom Neumann for insightful comments and discussions.

\section{References}

Billings, S.E., Kattenhorn, S.A., 2005. The great thickness debate: ice shell thickness models for Europa and comparisons with estimates based on flexure at ridges. Icarus 177, 397-412.

Brunt, K.M., 2008. Tidal motion of the Ross Ice Shelf and its interaction with the Siple Coast ice streams, Antarctica. Ph.D. thesis. The University of Chicago.

Brunt, K.M., Macayeal, D.R., 2014. Tidal modulation of ice-shelf flow: a viscous model of the Ross Ice Shelf. J. Glaciol. 60, 221.

Brunt, K.M., Fricker, H.A., Padman, L., Scambos, T.A., O'Neel, S., 2010a. Mapping the grounding zone of the Ross Ice Shelf, Antarctica, using ICESat laser altimetry. Ann. Glaciol. 51 (55), 71-79.

Brunt, K.M., King, M.A., Fricker, H.A., Macayeal, D.R., 2010b. Flow of the Ross Ice Shelf, Antarctica, is modulated by the ocean tide. J. Glaciol. 56, 157-161.

Fricker, H., Bassis, J., Minster, B., MacAyeal, D., 2005. ICESat's new perspective on ice shelf rifts: the vertical dimension. Geophys. Res. Lett. 32 (23), L23S08.

Gaidos, E.J., Nimmo, F., 2000. Planetary science: tectonics and water on Europa. Nature $405,637$.

Geissler, P.E., Greenberg, R., Hoppa, G., McEwen, A., Tufts, R., Phillips, C., Clark, B. Ockert-Bell, M., Helfenstein, P., Burns, J., Veverka, J., Sullivan, R., Greeley, R., Pappalardo, R.T., Head, J.W., Belton, M.J.S., Denk, T., 1998. Evolution of lineaments on Europa: clues from Galileo multispectral imaging observations. Icarus 135 107-126.

Greeley, R., Sullivan, R., Coon, M.D., Geissler, P.E., Tufts, B.R., Head, J.W., Pappalardo R.T., Moore, J.M., 1998. Terrestrial Sea ice morphology: considerations for Europa. Icarus $135,25-40$.

Greenberg, R., Geissler, P., Hoppa, G., Tufts, B.R., Durda, D.D., Pappalardo, R., Head, J.W., Greeley, R., Sullivan, R., Carr, M.H., 1998. Tectonic processes on Europa: tidal stresses, mechanical response, and visible features. Icarus 135, 64-78.

Gudmundsson, G.H., 2011. Ice-stream response to ocean tides and the form of the basal sliding law. Cryosphere 5, 259-270.

Head, J.W., Pappalardo, R.T., Sullivan, R., 1999. Europa: morphological characteristics of ridges and triple bands from Galileo data (E4 and E6) and assessment of a linear diapirism model. J. Geophys. Res. 104, 24223-24236.

Hurford, T.A., Beyer, R.A., Schmidt, B., Preblich, B., Sarid, A.R., Greenberg, R., 2005. Flexure of Europa's lithosphere due to ridge-loading. Icarus 177, 380-396.

Hurford, T.A., Helfenstein, P., Hoppa, G.V., Greenberg, R., Bills, B.G., 2007. Eruptions arising from tidally controlled periodic openings of rifts on Enceladus. Nature 447, 292-294.

Kadel, S.D., Fagents, S.A., Greeley, R., Galileo SSI Team, 1998. Trough-bounding ridge pairs on Europa - considerations for an endogenic model of formation. In: Lunar and Planetary Institute Science Conference Abstracts. In: Lunar and Planetary Inst. Technical Report, vol. 29, p. 1078.

King, M.A., Makinson, K., Gudmundsson, G.H., 2011. Nonlinear interaction between ocean tides and the Larsen C Ice Shelf system. Geophys. Res. Lett. 38, 8501.

Lee, S., Pappalardo, R.T., Makris, N.C., 2005. Mechanics of tidally driven fractures in Europa's ice shell. Icarus 177, 367-379.

Leith, A.C., McKinnon, W.B., 1996. Is there evidence for polar wander on Europa? Icarus 120, 387-398.

Leonard, K.C., Tremblay, L.-B., MacAyeal, D.R., Jacobs, S.S., 2008. Interactions of windtransported snow with a rift in the Ross Ice Shelf, Antarctica. Geophys. Res. Lett. 35 (5), L05501.

Makinson, K., King, M.A., Nicholls, K.W., Gudmundsson, G. Hilmar, 2012. Diurnal and semidiurnal tide-induced lateral movement of Ronne Ice Shelf, Antarctica. Geophys. Res. Lett. 39, 10501.

McEwen, A.S., 1986. Tidal reorientation and the fracturing of Jupiter's moon Europa. Nature 321, 49-51.

Padman, L., Erofeeva, S., Joughin, I., 2003. Tides of the Ross Sea and Ross Ice Shelf cavity. Antarct. Sci. 15 (1), 31-40.

Pappalardo, R., Coon, M.D., 1996. A sea ice analog for the surface of Europa. In: Lunar and Planetary Institute Science Conference Abstracts. In: Lunar and Planetary Inst. Technical Report, vol. 27, p. 997.

Porco, C.C., Helfenstein, P., Thomas, P.C., Ingersoll, A.P., Wisdom, J., West, R., Neukum, G., Denk, T., Wagner, R., Roatsch, T., Kieffer, S., Turtle, E., McEwen, A., Johnson, T.V., Rathbun, J., Veverka, J., Wilson, D., Perry, J., Spitale, J., Brahic, A., Burns, J.A., DelGenio, A.D., Dones, L., Murray, C.D., Squyres, S., 2006. Cassini observes the active South Pole of Enceladus. Science 311, 1393-1401.

Prockter, L.M., Patterson, G.W., 2009. Morphology and evolution of Europa's ridges and bands. In: Pappalardo, Robert T., McKinnon, William B., Khurana, Krishan K. 
(Eds.), Europa. In: The University of Arizona Space Science Series. University of Arizona Press, Tucson, ISBN 9780816528448, p. 237.

Prockter, L.M., Head, J.W., Pappalardo, R.T., Sullivan, R.J., Clifton, A.E., Giese, B., Wagner, R., Neukum, G., 2002. Morphology of Europan bands at high resolution: a mid-ocean ridge-type rift mechanism. J. Geophys. Res., Planets 107. http://dx.doi.org/10.1029/2000JE001458.

Rhoden, A.R., Wurman, G., Huff, E.M., Manga, M., Hurford, T.A., 2012. Shell tectonics: a mechanical model for strike-slip displacement on Europa. Icarus 218, 297-307.

Rignot, E., Jacobs, S., Mouginot, J., Scheuchl, B., 2013. Ice shelf melting around Antarctica. Science 341, 266.

Rignot, E., Mouginot, J., Scheuchl, B., 2011. Ice flow of the Antarctic ice sheet. Science 333 (6048), 1427-1430.

Scambos, T., Haran, T., Fahnestock, M., Painter, T., Bohlander, J., 2007. MODIS-based Mosaic of Antarctica (MOA) data sets: continent-wide surface morphology and snow grain size. Remote Sens. Environ. 111 (2), 242-257.

Schenk, P.M., McKinnon, W.B., 1989. Fault offsets and lateral crustal movement on Europa - evidence for a mobile ice shell. Icarus 79, 75-100.
Smith-Konter, B., Pappalardo, R.T., 2008. Tidally driven stress accumulation and shear failure of Enceladus's tiger stripes. Icarus 198, 435-451.

Spencer, J.R., Pearl, J.C., Segura, M., Flasar, F.M., Mamoutkine, A., Romani, P., Buratti, B.J., Hendrix, A.R., Spilker, L.J., Lopes, R.M.C., 2006. Cassini encounters Enceladus: background and the discovery of a South Polar Hot Spot. Science 311, 1401-1405.

Spitale, J.N., Porco, C.C., 2007. Association of the jets of Enceladus with the warmest regions on its south-polar fractures. Nature 449, 695-697.

Stempel, M.M., Barr, A.C., Pappalardo, R.T., 2005. Model constraints on the opening rates of bands on Europa. Icarus 177, 297-304.

Sullivan, R., Greeley, R., Homan, K., Klemaszewski, J., Belton, M.J.S., Carr, M.H., Chapman, C.R., Tufts, R., Head, J.W., Pappalardo, R., Moore, J., Thomas, P., 1998. Episodic plate separation and fracture infill on the surface of Europa. Nature $391,371$.

Tufts, B.R., Greenberg, R., Hoppa, G., Geissler, P., 2000. Lithospheric dilation on Europa. Icarus 146, 75-97.

Turtle, E.P., Melosh, H.J., Phillips, C.B., 1998. Tectonic modeling of the formation of Europan ridges. EOS Trans. AGU 79, F541. 